\title{
Uterus bicornis septus; Vagina partim septa; Atresia vaginae dextrae congenita consecutiva; Haematometra dextra; Haematosalpinx dextra; Haematocolpos; Punctio haemato- metrae; exitus letalis.
}

\author{
Mitgetheilt aus der Dresdener Entbindungsanstalt. \\ Von
}

Dr. P. Näcke.

Die Zahl der bereits veröffentlichten Fälle von einseitiger Haematometra bei Verdoppelung des Genitalkanales ist eine verhältnissmässig noch kleine, so dass $\mathrm{Schr}$ öd er in seinem Lehrbuche der Gynäkologie (1875) nur von 34 klinisch beobachteten Fällen spricht (S. 55). Es dürfte daher die Mittheilung des folgenden Falles, der in mehr als einer Hinsicht Interesse darbietet, als ein weiterer Beitrag erwünscht sein.

Emilie Bauer, 20 Jahre alt, aus Kleinneuschönberg bei 01bernhau, ward in die Krankenstation des Dresdener Entbindungsinstitutes den 24. Juni 1874 aufgenommen.

Anamnese. Eltern leben noch; Patientin ist, bis auf Scharlach im Kindesalter und Erysipelas faciei im 19. Jahre, immer gesund geblieben. Erste Regel im 15. Jahre; von da ab bestand sie circa zwei Jahre lang sehr unregelmässig, kehrte zwischen 3-7 Wochen wieder; Dauer nicht zu ermitteln; immer viel Blutverlust. Die Regel ging mit so heftig auftretenden Kreuz-, Leib- und Kopfschmerzen einher, sowie allgemeiner Mattigkeit, dass Patientin meist acht Tage lang zu Bett liegen musste, während der Blutabgang selbst nicht so lange dauerte. Die Leibschmerzen wurden nur in der rechten Seite empfunden. Vom 17. Jahre ab ward die Menstruation regelmässiger, aller 3-4, manchmal 5 Wochen, 2-3 Tage dauernd. Immer viel Leibschmerzen und allgemeine Mattigkeit vom 3. Tage vor bis zwei Tage nach der Regel, Beschwerden, die Patientin gleichfalls öfter zwangen, acht Tage lang das Bett zu hüten. Sie giebt auch als besonders unangenehm ein andanerndes, intensives Hitzgefühl in den Genitalien an. Die Regel besteht bis jetzt ununterbrochen fort; letzte den 17. Februar 1874.

Einige Zeit nach der ersten Regel bemerkte Patientin über dem rechten Ligamentum Poupartii eine Geschwulst in Hühnereigrösse (eher gar nicht bemerkt), die anfangs für zurückgehaltenes Blut angesehen wurde und sowohl ausserhalb der Regel als während derselben - nur hier heftiger und andauernder - schmerzte. Die Geschwulst wuchs immer mehr heran; ob immer blos während der 
Menses, ist nicht zu ermitteln. Immer aber hat Patientin, sobald die Schmerzen eintraten, das Gefühl des Grösserwerdens des Tumors. Später ward der Tumor für eine Cyste gehalten. Oppressionserscheinungen fehlen; bisweilen Uebelkeit, kein Erbrechen.

Harn und Stahl werden nur während des Bestehens dex Schmerzen schwer entleert; indess besteht schon seit einiger Zeit eine geringe Neigung zu Verstopfung.

Status praesens. Mittelgrösse; Blondine; ziemlich rothe Backen; Haut locker angeheftet, nicht besonders fettreich; kräftige Muskulatur und Knochenbau.

Thorax normal. Die normal entwickelten Brüste liefern auf Druck kein Secret.

Leib Hach, zeigt nur dicht über dem rechten Ligamentum Poupartii eine sehr deutliche, gänseeigrosse, bei der Respiration unbewegliche Geschwulst, über weleher die Haut nicht geröthet, leicht versehieblich, nicht besonders gespannt ist. Sie reicht bis dicht an die Mittellinie; nach aussen endet sie $21 / 2 \mathrm{Cm}$. von der Spina anterior superior dextra. Sie ist $4 \mathbf{1} / \mathbf{2} \mathrm{Cm}$. hoch, 5 breit und geht nach hinten in eine schmale, bis zur Nabelhöhle reichende, mehr durch das Gefühl als durch das Gesicht wahrnehmbare Geschwulst iiber. Die Spitze derselben kann man durch die Percussion sicher von der Lueber abgrenzen, durch einen $2 \mathrm{Cm}$. breiten, tympanitischen Ton., Ebenso lässt sich die. Milz percutorisch sicher nachweisen.

Bei der Palpation findet man in der tiefer liegenden Geschwulst prallelastische, fluctuirende Consistenz; die höher hinauf gelegene ist nur als prallelastisch zu bezeichnen. Vulva geschlossen, bis zum Damm stark behaart; Nymphen stark prominent. Hymen intact, Rand etwas gefranzt. Scheide lang, weit, glatt. Urin klar, ohne Albumin.

In der Scheide fühlt man nahezu in der Mittellinie, aber ziemlich hochstehend, einen Muttermund, der eben für die Fingerspitze durchgängig ist. Die vordere Scheidenwand und das vordere Scheidengewölbe sind etwas herabgedrängt und zeigen gleichfalls prallelastische, fluctuirende Beschaffenheit. Bei der bimanuellen Untersuchugg stellt sich heraus, dass beide Abschnitte des oben beschriebenen Tumors mit der Geschwulst in der Scheide zusammenhängen, dass ein Druck auf das eine Ende der Geschwulst deutlich am anderen Ende gefühlt wird, endlich dass die tiefer liegende Gesehwulst ziemlich innig mit der vorderen Beckenwand verwachsen ist. Der Tumor in der Scheide setzt sich unmittelbar, ohne einen Scheidentheil hervortreten zu lassen, in den Muttermund fort und erscheint deshalb der Uterus, der wegen des im Ganzen wenig bewegliehen, nicht zu umgreifenden Tumors für die bimanuelle Untersuchung nicht zugänglich ist, fest mit der Geschwulst verwachsen. Bei der Untersuchung per anum in der Narcose kann nan wegen des geringen Spielraumes nur mit der halben Hand bis zu den. Mittelhand- 
knochen eindringen und ist neben dem grossen Tumor undeutlich ein kleiner, länglich ovaler Körper zu fühlen. (Durch die Untersuchung entstehen zwei kleine Fissuren:)

In Anbetracht nun der Art und Weise der Entstehung, sowie des weiteren Wachsthumes des Geschwulst bei fortdauernder Menstruation; in Anbetracht der Lage derselben, insofern sie in dem oberen Theile nicht ganz, in ihrem unteren gerade bis an die Mittellinie heranreicht, mithin auf die rechte Körperhälfte beschränkt ist; in Anbetracht ihrer fluctuirenden Consistenz, die die Fortpflanzung der Wellen ermöglicht; endlich mit Rücksicht darauf, dass der Scheidentheil verstrichen ist, wird unter Ausschluss einer Ovarieneyste mit ganz kurzem Stiele, von Prof. Dr. Winckel die Diagnose auf Uterus bicornis, Haematometra der rechten Hälfte. Haematosalpinx und Haematocolpos (das letztere noch reservirt) gestellt, Bei der darauf vorgenommenen Probepunction quillt eine zähe, braune, theerartige Flüssigkeit aus dem Troicar heraus.

Weiterer Verlauf. Den 26. Juni. Bei niedriger Temperatur geringe Schmerzen am Anus (Fissuren). Vormittags $11 \mathrm{Uhr}$ wird der Tumor von der Vagina ans, eirca $4 \mathrm{Cm}$. unterhalb des Muttermundes, punctirt und werden 22,0 dicken, syrupartigen Blutes durch die Caniule entleert. Die Flüssigkeit schwimmt auf dem Wasser; mikroskopisch sieht man, wie gestern, zahlreiche, sternförmige, rothe Blutkörperchen neben intacten; viel Detritus, keine Haematinkrystalle. Abends Schmerzen im Epigastrium. Nachmittags 4 Uhr ist die mittlerweile abgeflossene, zähe Flüssigkeit gewogen, und da die Canüle nichts mehr ausfliessen liess, die letztere mit einem Wattepfropf verschlossen worden. Gewicht der Flüssigkeit 73,0. Die Patientin wird nach Verschluss der Canüle vorsichtig vom Operationstische in das Krankenbett gebracht.

Den 27. Juni. Temperatur 37,0, 92 Puls. Schmerzen mehr im Epigastrium. Catheterisiren. Kein Appetit; Zunge belegt, etwas gesehwollen: sehr viel Durst. Beim Herausnehmen des Wattepfropfes fliesst eine dünne, schwach nach $\mathrm{SH}$ riechende Flüssigkeit $\mathrm{ab}$, nur der Bodensatz ist zäher. Gewicht des in circa $1 / 4$ Stunde bis zum Versiegen abgelaufenen Fluidums beträgt 62,0.

Die andere Geschwulst erscheint flacher, hat jedoch an Grösse und Resistenz noch nicht abgenommen; nur die obere hintere ist in ihrem Umfange bedeutend verkleinert. Die Diagnose wird jetzt mit Sicherheit auch auf Haematocolpos gestellt. Patientin entleert im Laufe des Tages $80 ; 0$ einer braunen, absetzenden, unten zähen, iu den oberen Schichten helleren blutserumähnlichen Flüssigkeit. Abends wird die Canüle wieder mit einem Korkstöpsel verschlossen. Befinden sonst gut.

Den 28. Juni. Temperatur 37,4, Puls 92. Leidlich geschlafen. Gegen Morgen empfand Patientin plötzlich stechende Schmerzen in der Gegend der rechten Tube, die jedoch bald wieder nachlassen. Die obere Geschwulst fast ganz verschwumden, die untere noch ziemlich prall gefüllt; über derselben schwach tympanitischer Ton. Leib 
nirgends schmerzhaft; nur bei stärkerem Drucke über der ehemaligen oberen Geschwulst. Wasser spontan gelassen. Canüle ist herausgerutscht; Muttermund ist etwas tiefer getreten; neben demselben jedoch noch eine bedeutenide Hürte fühlbar. Einlegen eines elastischen Catheters. Mittags 12 Uhr klagt Patientin uber heftige Schmerzen in der Gegend der rechten Tube, die bei Druck sich steigern. Morphium subcutan 0,01. Puls 100, voll. Abends 7 Uhr: 38,3 und 100 Pulse. Schmerzhaftigkeit des Leibes mehr zu- als abgenommen. 0,01 Morphium subeutan. Abends 11. Uhr: 116 Pulse, 68 Resp., 2 Mal erbrochen. Hände etwas kühl, Puls klein. Leib schwach meteoristisch anfgetrieben, links hart anzufühlen. Links in der Seite etwas Dämpfung. (Während des vergangenen Tages noch 30,0 dünne Flüssigkeit entleert.) Eisblase.

Den 29. Juni. Temperatur 37,8; Puls 160 , Respiration 80. Athem frequent; Leber $3 \mathrm{Cm}$. über dem Rippenrande. $1 \mathrm{Mal}$ Stuhl. Nach demselben Befinden etwas besser, 140 Pulse, 60 Respirationen. Gesichtsausdruck ruhig. Extremitäten kühl. Patientin liegt nach rechts gewandt und klagt über Schmerzen unter beiden Rippenbögen und oberhalb des Nabels. Die Gegen'd der ursprünglich am meisten prominirenden Geschwulst ist sehr abgeflacht, und man kann jetzt, obwohl man die Contouren derselben noch abzugreifen vermag, bis. dicht über das Ligamentum Poupartii tympanitischen Ton verfolgen. Viel Durst, gegen Morgen drei Mal erbrochen. Mässiges Exsudat im Leibe. Pulvis Doveri drei Mal täglich. Nachmittags 2 Uhr 30 Minuten Befinden besser; eatheterisirt. Abends 38,4 Temperatur und 160 Pulse. Viel Durst, sonst keine Klagen. Catheterisirt. Patientin bei Sinnen. Aus der Canüle ist während des Tages eine kaum nennenswerthe Quantität Fliissigkeit ausgesickert.

Den 30. Juni. Temperatur 38,4 und 152 Pulse, 44 Respirationen. Gesichtsausdruck wechselt, heftiges Athmen; Meteorismus hat zugenommen; kühle Extremitäten; wenig geschlafen; einige Male delirirt; Harn unter sich gehen lassen. Mehrmals Erbrechen. Bei der Visite ist der Kopf frei, die Antworten klar. 4 Uhr Nachmittags: Agone. 1/2, Stunde später Tod.

Dies die Krankengeschichte, dem Krankenjournale entnommen. Die durch Dr. Birch-Hirschfeld ausgeführte Section ergiebt in der Hauptsache Folgendes:

In der Trachea reichlich schaumiges Fluidum. Lungen überall ödematös; in den Lungengefässen flüssiges Blut. Pleurahöhlen, Herz normal: Die Bauchhöhle enthält etwa 500,0 ziemlich dünner, braunrother, übelriechender, freier Flüssigkeit.

Die serösen Häute sind vielfach unter einander verklebt, von mehr oder weniger ausgebreiteten schmutziggelbbraunen Pseudomembranen bedeckt; solche befinden sich namentlich auf der vorderen Fläche der Leberkapsel und auf der Serosa der Genitalorgane. Leber ziemlich normal; Milz etwas vergrössert, auf der Kapsel dicke Fibrinmembran. An beiden Nieren sind Becken und Ureteren etwas ausgedehnt, besonders rechts, und die Pyramiden etwas abgeflacht. 
Magen normal. Dünndarm im Allgemeinen aufgetrieben, seine Serosa vielfach verklebt, die Serosagefässe lebhaft injicirt. Der Dickdarm ebenfalls aufgetrieben. Schleimhaut normal.

Nach Entfernung der Därme ist der grösste Theil des Beckens, namentlich von rechts her, eingenommen durch. eine rechts bis über den Hüftkamm reichende Cyste, deren äussere Wand einerseits mit strangartigen Partien des Omentum, andererseits mit dem Peritonealüberzuge der vorderen Bauchwand, dem S-Romanum, und nach vorn mit dem rechten Uterushorne (s. d.) innig verklebt und verwachsen ist. Bei Druek auf diese Cyste, deren Wand bereits von aussen schwärzlich gefärbt erscheint, entleert sich aus derselben eine ähnliche, fast chocoladenfarbige Flüssigkeit, wie sie sich frei in der Bauchhöhle fand, und zwar dringt dieses Fluidum aus einer kleinen Oeffnung hervor, welche sich an der oberen und rechten Grenze der Cyste befindet; es hat sich hier, während die Nachbarschaft der Perforationsstelle noch innig mit der Bauchwand zusammenhängt, eine etwa linsengrosse Partie von derselben losgelöst, und es ist dadurch die erwähnte Perforation der Cystenwand entstanden.

Während nun das linke Horn des Uterus frei (nur bedeckt von missfarbigen Pseudomembranen) nach links hinüberliegt, in Verbindung mit der zugehörigen ziemlich weiten Tube und dem Ovarium, welches méhrfache mit dünner fadenziehender Flüssigkeit erfüllte Cysten enthält, befindet sich das rechte Horn (welches an seiner Basis nach links hin mit dem linken lediglich durch eine Bauchduplicatur zusammenhängt) vor der erwähnten Cyste; mit deren vorderer Wand seine Hinterfläche auf das Innigste zusammenhängt; es wird dieses Horn aber auch von rechts her von dieser sich nach dieser Seite hin umbiegenden Cyste umgriffen, während vorn und rechts da, wo das letzte etwas verjüngte Ende der letzteren dem Uterushorne anliegt, sich das in hohem Grade cystös degenerirte rechte Ovarium befindet; die Cysten, welche die Substanz des letzteren fast völlig ersetzen, enthalten ebenfalls eine ziemlich dünne Flüsssigkeit.

Beim Aufschneiden der mehrfach erwähnten Cyste präsentirt sich ein (wie angegeben) von links wach rechts verlaufender und dann nach dem rechten Uterushorn umbiegender, weiter Hohlraum, welcher jedoch dadurch einigermaassen fächrig erscheint, dass von seiner Innenfläche her sich in grösseren Abständen lamellöse Vorsprïnge in das Innere der Cyste hinein erheben; dieselben sitzen quer zum Verlauf des Cystenkanales, sie springen meist nur $1-2 \mathrm{Cm}$. vor, so dass nirgends eine völlige Abtheilung eingetreten ist. Die Cyste enthält ausser der erwähnten chocoladenfarbigen Flüssigkeit reichliche, festere, bröckliche, bräunliche Massen, welche zum Theil der Innenfläche innig anhaften; letztere ist im Allgemeinen ziemlich rauh, höckerig; sie enthält übrigens nirgends secundäre Cysten. Der Stelle entsprechend, wo sich am rechten Uterushorne die Tube ansetzen. müsste, ist, wie erwähnt, die Cyste trichterförmig verjüngt, doch besteht keine Communication mit der Uterushöhle. 
Das rechte Uterushorn ist über noch ein Mal so gross als das erwähnte linke; seine Wandung ist fast von weissglänzender Farbe, beiläufig $1 \mathrm{Cm}$. dick; in der Höhle finden sich noch geringe Mengen krïmlicher, braungelber Blutcoagula; die Mucosa erscheint rauh durch Auflagerung, stellenweise wie angenagt, von schwärzlicher Farbe; der Cervicalkanal ist deutlich kenntlich, ebenso die Portio vaginalis; das Orificium mündet in ein etwa apfelgrosses Cavum, in welchem sich noch ebensolehe veränderte Blutcoagula wie in der Uterushöhle, vorfinden. Durch eine (Punctions-) Oeffnung communicirt die Höhle dieses rechtsseitigen Scheidenrudimentes mit der línken Vagina, während eine natürliche Communication sich nicht vorfindet. Von der linken Scheide aus gesehen, liegt die erwähnte rundliche, etwa bleistiftdicke Punctionsöffnung im Centrum einer etwa markgrossen, in die rechte Wand der linken Vagina sich vorbuchtenden Partie, welche sich derber anfühlt. Die Oeffnung liegt fast $5 \mathrm{Cm}$. oberhalb des einfachen, dilatirten Hymens der linken Scheide; die letztere verläuft oberhalb und nach links von der erwähnten Punctionsstelle noch in der Länge von $4 \mathrm{Cm}$; ein Scheidengewölbe ist an derselben kaum angedeutet, ebenso die Portio vaginalis des linken Uterushornes, dessen Orificium als eine linsengrosse Oeffunng sich darstellt. Die Innenfläche des linken Uterushornes bietet nichts Abnormes.

Die Harnblase ist mit der rechten Hälfte ihren Hinterfläche mit dem Uterushorne innig verwachsen. Sonst ziemlich normal. An den äusseren Genitalien ist nichts Abnormes.

Als Ergänzung zu voranstehendem Sectionsprotocolle mögen folgende Benerkungen dienen, welche eine genaue Untersuchung des fraglichen Spirituspräparates veranlasst hat.

Die hintere Blasenwand zeigt aussen viele Auflagerungen; membranöse Stränge gehen von hier hinab zum-linken Uterushorne und zeigen mehrere Unterbrechungen in Form von kreisrunden Löchern. Das Ligamentum vesico-uterinum ist gut entwickelt und geht zum Mittelstïck des Uterus. Das letztere hat als grössten Durchmesser von vorn nach hinten eine Länge von $3,5 \mathrm{Cm}$., bei fast gleicher Breite, und senkt sich stark gewölbt nach hinten tief in den Douglas'schen Raum ein. Die hintere Fläche ist fast ganz glatt und zeigt gerade genau in der Mitte eine circa $3 \mathrm{Cm}$. lange, deutliche flache Furche. Das Cavum Douglasii ist tief, aber sehr schmal und ist von vielen kleinen Membranen durchsetzt, und reicht bis etwa ${ }_{1 / 2} \mathrm{Cm}$. unterhalb des Orificium externum des linken Uterushornes. Das linke Uterushorn misst vom Os uteri internum bis zum Fundus aussen $5 \mathrm{Cm}$; grösste Breite $2^{2 / 3}$. Hintere Wand $1 \frac{1}{3} \mathrm{Cm}$. dick, vordere weniger dick. Cavum uteri $2,25 \mathrm{Cm}$. lang, Fundusstelle $1 \% / 8$ Cm. dick. An der hinteren $W$ and viele Auflagerungen and ganz dünne, aber breite Stränge zum linken Ovarium. Die Längsachsen beider Uterushörner liegen am Präparate ziemlich in einer Richtung, dem queren Durchmesser des Beckens entsprechend. Auf der linken Seite liegen alle Gebilde des Ligamentum latum klar und deutlich vor. 
Das rechte Uterushorn misst vom Os uteri internum bis zum Fundus aussen $6^{1 / 3} \mathrm{Cm}$. Wände $1 \frac{1}{3} \mathrm{Cm}$. dick. Cavum uteri $4,5 \mathrm{Cm}$., Collum $1^{2 / 3}$, leicht gebogen mit der Concavität nach rechts aussen. Vom Fundus kann man mit einer Sonde die rechte Tube auf eine Strecke von circa $2,5 \mathrm{Cm}$. verfolgen; dann ist der Kanal obliterirt und der übrige Tubentheil bildet die frühere besehriebene cystöse Ausweitung. Das rechte Ligamentum latum ist in toto "so verfilat und mit der Umgegend verwachsen, dass man nicht einmal das Ovarium sehen, wohl aber fühlen kann.

Die aufgeschnittene Tubencyste hat ausgebreitet die Gestalt eines Blattes eines Weinstockes; die grösste Länge beträgt $11^{2 / 3}$, die grösste Breite 121/4 Cm. Die Wandungen nach hinten und rechts sind 0,25 dick, dägegen links und vorn nur die Hälfte. Es kann keine Communication mit der Tube entdeckt werden.

Starke Querfalten sind blos in der vorderen Hälfte der Scheide. $4 \mathrm{Cm}$. vom Hymen ab senkt sich von der oberen Wand ein etwa $4 \mathrm{Cm}$. langer (von vorn nach hinten), $3^{3} /{ }_{4} \mathrm{Cm}$. breiter (von rechts nach links) und etwa $1,5 \mathrm{~cm}$. tief in die Scheide herabreichender, überall glatter und gleich convexer Tumor. Etwa in der Mitte, fast ganz die hintere Vaginalwand berührend, befindet sich die $2 / 3 \mathrm{Cm}$. grosse Punctionsöffnung, deren Entfernung vom Os uteri internum des rechten Uterushornes $4,5 \mathrm{Cm}$. beträgt. Das Collum uteri ist ganz eng, auch das Cazum uteri kaum ausgedehnt zu nennen. Zwischen beiden Hörnern des Uterus schiebt sich in der ganzen Länge seines Mittelstückes das rechte Scheidenrudiment ein und zwar in Gestalt eines fast rechtwinkeligen Dreieckes, deren Hypotenuse nach der linken Scheide, die gegenüberliegende Cathete nach dem Kreuzbeine zu liegen.

Von der Punctionsöffnung nach hinten $2^{3 / 4} \mathrm{Cm}$. entfernt, befindet sich der circa $1^{1 / 2} \mathrm{Cm}$. lange, etwas klaffende Muttermund des linken Hornes. Das Collum uteri des letzteren beträgt $3 \mathrm{Cm}$. Die dazu gehörige Vaginalportion ist von dem Tumor durch einen nur $1 / 3 \mathrm{Cm}$. vorspringenden Wulst geschieden.

Interessant ist in unserem Falle die Angabe, dass während der Menstruation Schmerzen nur rechts in der Inguinalgegend angegeben wurden und jedenfalls mit der in diese Zeit fallenden periodischen Vergrösserung des Tubentumors zusammenhingen. Dass letztere nicht der Rï̈kstauung des Blutes den Ursprung verdankte, sondern der Blutausscheidung aus der Tubenschleimbaut, wird in unserem Falle durch die fehlende Communication zwischen Tube und Uterus, die vielleicht erst nur secundär eintrat, bewiesen. Das aus der ursprünglich offen gewesenen Tubenöffnung aussickernde Blut hat jedenfalls durch Reizung der Serosa die vielfachen Verklebungen der Beckenorgane, die Pseudomembranen, und schliesslich die Verlöthung der Tubenöffnnng mit der vorderen Bauchwand bewirkt. Denn die später auftretende Perforationsperitonitis war von zu kurzer Dauer, um solche weitgehende Veränderungen zu setzen. Die Zerreissung des Blutsackes nach der Punction der Haematometra [durch plötzlich, $1 \frac{1}{2}$ 'Tage 
etwa nach der Operation, auftretenden Schmerz in der Tubengegend und fast vollkommenes Verschwinden des Tumors charakterisirt] ist durch Verminderung des Luftdruckes im Abdomen nach Entlastung des Uterus entstanden; doch mögen Contractionen des Uterus und die Báuchpresse durch Zerren an den Adhäsionen auch mit dazu beigetragen haben. Ich möchte mich daher auch jenen französischen Operateuren (Dupuytren, Cazeaux) anschliessen, die bei diagnostieirter Haematosalpinx die Punction der Haematometra lieber zu unterlassen vorschlagen.

Der Tod ist in unserem Falle der Perforationsperitonitis und der Resorption des zersetzten Inhaltes des Uterus zuzuschreiben.

Sehr interessant ist endlich auch das vollkommene Zurücktreten der Haematometra [Uterushöhle nư gering vergrössert] gegen die Haematosalpinx und Haematocolpos. Von Spiralstellung beider Scheiden, wie auch von Längsfalten der Scheide als Reste des Septums zwischen den Müller'schen Gängen, konnte man nichts sehen.

\section{Ueber Cysten des breiten Mutterbandes.}

$$
\text { Von }
$$

\section{A. Gusserow. ${ }^{1}$ )}

Während die pathologisch-anatomischen Untersuchungen der Ovariengeschwiuilste schon seit langer Zeit gelehrt haben, dass die meisten sogenaninten einfächerigen Cysten der Ovarien doch in die Classe der zusammengesetzten Kystome gehören, und dass der Hydrops folliculi sehr selten eine erhebliche Grösse erreicht, sondern meist nur kleine Tumoren ohne Bedeutung für Leben und Gesundheit

1) Nachfolgender kleiner Aufsatz war bereits seit Sommer 1875 druckfertig und seine Publication wurde von mir nur hinausgeschoben, um über den weiteren Verlauf des $\mathrm{zu}$ beschreibenden Kranksheitsfalles möglichst sicher urtheilen zu können. Mittlerweile ist nun im 1. Hefte dieses Bandes eine interessante Beobachtung und Besprechung eines gleichen Krankheitszustandes von Schatz erschienen. Bei der Wichtigkeit des Gegenstandes dürfte aber meine folgende Mittheilung doch noch am Platze sein. Ich habe auf den Fall von Sch atz nicht weiter recurrirt, weil derselbe ja den Lesern dieses Archirs hinreichend bekannt ist. Nur in meinen Literaturangaben habe ich wesentlich gekürzt, weil die vorhandene Literatur von Schatz nahezu erschöpfend benutzt ist. 